\title{
Hierarchical Patch Generation for Multi-level Statistical Shape Analysis by Principal Factor Analysis Decomposition
}

\author{
Mauricio Reyes ${ }^{1}$, Miguel A. González Ballester ${ }^{2}$, Nina Kozic ${ }^{1}$, Jesse K. Sandberg ${ }^{3}$, \\ Ronald M. Summers ${ }^{3}$, Marius George Linguraru ${ }^{3}$ \\ ${ }^{1}$ ARTORG Center for Biomedical Engineering Research, University of Bern, Switzerland \\ ${ }^{2}$ Alma IT Systems, Barcelona, Spain \\ ${ }^{3}$ Radiology and Imaging Sciences, Clinical Center, National Institutes of Health, \\ Bethesda, MD, USA \\ lingurarum@mail.nih.gov
}

\begin{abstract}
We present a framework for multi-level statistical shape analysis, applied to the study of anatomical variability of abdominal organs. Statistical models were built hierarchically, allowing the representation of different levels of detail. Principal factor analysis was used for decomposition of deformation fields obtained from non-rigid registration at different levels, and provided a compact model to study shape variability within the abdomen. To assess and ease the interpretability of the resulting deformation modes, a clustering technique of the deformation vectors was proposed. The analysis of deformation fields showed a strong correlation with anatomical landmarks and known mechanical deformations in the abdomen. Clusters of modes of deformation from fine-to-coarse levels explain tissue properties, and inter-organ relationships. Our method further presents the automated hierarchical partitioning of organs into anatomically significant components that represent potentially important constraints for abdominal diagnosis and modeling, and that may be used as a complement to multi-level statistical shape models.
\end{abstract}

Keywords: statistical models, shape decomposition, principal factor analysis, hierarchical clusterization, multi-organ variability, anatomical reconstruction.

\section{INTRODUCTION}

Statistical atlases of the abdomen represent useful tools for the initialization of segmentation of organs [7]. In a more comprehensive manner, multi-level statistical shape models [9] have been proposed to allow better shape representation of complex structures, and to add more flexibility in segmentation procedures. Their approach is to consider initially the whole shape, and then define lower levels by subdividing it iteratively into $N$ patches. At each level, a Principal Component Analysis (PCA) of shape is performed to define more detailed localized variations at each successive level. Adhesiveness constraints are imposed in order to guarantee geometrical continuity between neighboring patches. This approach was successfully applied to the segmentation of the liver in CT images [9]. The approach was further applied to the simultaneous segmentation of multiple organs within the abdominal cavity, thus extending the concept to multiorgan statistical shape models [8]. In these methods, the subdivision of the shape into patches is defined manually without obvious anatomical knowledge about the organ under consideration. 
Partitioning of shapes for shape analysis has also been tackled by Syrkina et al [13], this time for statistical modeling of shapes with arbitrary topology. While they use an automatic optimization process for the definition of the patches, which guarantees the topology and uniformity of each patch, initialization of the patches is based on uniform spreading of seed points across the surface. Thus, no knowledge derived from the anatomy, physiology or shape variability is used. Our approach could be applied as a principle patch generation procedure.

In general, statistical shape analysis techniques commonly employed in the medical imaging community, such as Active Shape Models or Active Appearance Models rely on Principal Component Analysis (PCA) to decompose shape variability into a reduced set of interpretable components [2,4]. In this paper we use Principal Factor Analysis (PFA) as an alternative to PCA, as it is potentially better suited for medical imaging applications, from the point of view of interpretability of the shape decomposition [5].

Additionally, we propose a method for automatic partitioning of shapes in the framework of statistical shape modeling, based on criteria derived from a PFA analysis, which is shown to correlate to the inherent anatomy and physiology, while also incorporating knowledge derived from the shape variability found in the datasets. For patch generation, we cluster a vector field describing the mode of deformation across a surface, which is based on the minimization of a twoterm energy: a first term based on the co-linearity among vector directions and a second term that considers the area gain when adding a candidate point to a cluster. Patches are generated automatically from coarse to fine levels by varying the clusterization constraints.

Our approach could be applied as a principle patch generation procedure. The results are shown to correlate to inherent abdominal anatomy and physiology, while also incorporating knowledge derived from the shape variability found in the dataset. This echnique offers an automated hierarchical scheme to build multi-level (potentially multi-organ) statistical models of the abdomen.

\section{METHOD}

\subsection{Data}

Ten abdominal CT scans of patients with no abnormalities in the ten studied organs were used: 5 male and 5 female (mean age of 59.9 years: 60.6 for male and 59.2 for female). Data were collected with a LightSpeed Ultra scanner (GE Healthcare) and image resolution ranged from $0.54 \times 0.54 \times 1.0 \mathrm{~mm}^{3}$ to $0.77 \times 0.77 \times 1.00 \mathrm{~mm}^{3}$. The liver, spleen, left kidney, right kidney, left adrenal gland, right adrenal gland, gallbladder, pancreas, stomach and aorta were manually segmented in the $10 \mathrm{CT}$ scans and masks of the organs were generated.

\subsection{Model Construction}

For the construction of the models, a random image from the database is set as reference and all other subject data are registered to the reference. For all subjects, the manual segmentation of ten abdominal organs (liver, spleen, kidneys, pancreas, stomach, gallbladder, adrenal glands and aorta) was performed and masks of the organs were generated. Organ coordinates in each subject were normalized relative to the position of the xiphoid to reduce variability from image acquisition. Then registration was performed employing the non-linear algorithm based on B-splines [12] and normalized mutual information [14]. Each organ of each subject was registered individually to its corresponding mask in the reference.

The deformation of objects is governed by an underlying mesh of control points in a coarse to fine multiresolution approach. B-splines allow to locally controlling the deformation and a compromise between the similarities provided by the mutual information and smoothing is searched. The resulting deformation fields between the reference image and the subject data are input in the analysis of anatomical variation. Once point correspondences are established for every organ and for every sample dataset, anatomical variability is modeled through PFA. 


\subsection{Principal Factor Analysis}

Principal component analysis (PCA) is a projection model for factor analysis (FA) that aims to find a low-dimensional manifold in the space of the data, such that the distance between the data and its projection on the manifold is small [1]. PCA is the best, in the mean-square error sense, linear dimension reduction technique [4]. Unlike the popular PCA, PFA can be considered as a generative model for FA. Generative models try to model the density function that is assumed to have generated the data, under a set of constraints that restricts the set of possible models to those with a low intrinsic dimensionality [1]. Whereas PFA models covariance between variables, PCA models the total variance in the data and as such it determines the factors that account for the total (unique and common) variance in the set of variables. Contrarily, PFA determines the least number of factors that can account for the common variance (correlation).

A common difficulty encountered when using PCA for shape analysis is that of correlating the resulting modes of variation with intuitive shape descriptions employed by clinical partners. Thus, these components are often described as combinations of several localized shears, twists, rotations, etc., but these are most often simplistic approximations to complex deformation fields. While PCA is recommended for dimensionality reduction, PFA is adapted for the study of structure in the data.

Details on the computation of PFA can be found in [5]. In this work, we use the expectation-maximization (EM) algorithm of Rubin [11], and employ varimax rotation [6] to fix the factor rotation.

\subsection{Clustering}

For patch generation, we clustered a vector field describing the mode of deformation across a surface. The result from the generation of the statistical models is a point distribution model (PDM). The PDM is able to describe the shape variability of the structure as a surface or point cloud embedded in the $3 \mathrm{D}$ space. Let $P=\left\{p_{1}, p_{2}, \ldots, p_{M}\right\}, M \in \mathrm{N}^{*}$, be a set of points that generate a surface in a domain $D \in \mathrm{R}^{3}$. For each $p_{i} \in P, i=1, \ldots, M$, a vector $V_{\mathrm{i}}$ is computed as

$$
\begin{aligned}
& V_{i}=v_{i}^{+}-v_{i}^{-}, \\
& \text {with } v_{i}^{+}=\bar{m}+\alpha_{j} \Phi_{j} \\
& \text { and } v_{i}^{-}=\bar{m}-\alpha_{j} \Phi_{j}
\end{aligned}
$$

$v_{i}^{+}$and $v_{i}^{-}$denote the generic PDM model generated from PFA, where $\bar{m}$ corresponds to the mean shape and $\alpha_{j}$ is a scaling value for the $j^{\text {th }}$ principal mode of the eigen-matrix $\phi_{j} . \alpha_{j}$ is chosen according to the plausible range of values that generate valid shapes within the PDM training dataset.

A clusterization across the surface was initially presented in [10], which was conducted for vector field segmentation of moving objects in 2D image sequences. We extend this work to unstructured 3D displacement vector fields across a surface. The clusterization process can be seen as a minimization problem of the following functional over a region or domain $\Omega \subseteq D$

$$
C=\arg \min _{\Omega} J(\Omega)
$$

where $J$ is an energy with two components: a first component takes into account the colinearity between vectors within the domain $\Omega$ and the predominant vector direction $\mathrm{V}_{\Omega}$ in $\Omega$, weighted by the vector length in order to give more importance to regions having a stronger deformation; the second term acts as a maximal area constraint.

$$
J(\Omega)=\int_{\Omega}\left(\frac{\left|V_{\Omega} \times V(m)\right|}{|V(m)|}\right)^{2} \frac{L_{\max }}{|V(m)|} d m+\gamma \int_{D \backslash \Omega} d m,
$$


where $\gamma$ is a real value and $L_{\max }=\max _{D}\{|V(m)|\}$. The dominant vector direction $V_{\Omega}$ is found as the highest eigenvalue of the following matrix

$$
M(\Omega)=\int_{\Omega} V(m) V^{t}(m) d m
$$

The minimization of Equation (2) is done by a hierarchical scheme, where each pattern is first considered as a cluster and then iteratively visited and agglomerated according to the energy measure $J$, until all points on the surface have been analyzed.

Patches were generated automatically from coarse to fine levels; the number of clusters is not pre-set in our application. Through functional minimization and the tradeoff between sparseness of the deformations and clusters size, the algorithm provides a clusterization of the $2 \mathrm{D}$ surface embedded in the $3 \mathrm{D}$ space (we emphasizes again the tailoring of this approach compared to [10]) with no preconditioning on the expected number of clusters. Our method was designed to assess the interpretability of the decompositions by PFA in conjunction with anatomical variability.

\section{RESULTS}

Patches were generated from the clusterization of deformation modes from PFA decomposition for ten abdominal organs. Examples are presented in Figures 1, 2 and 3 for the automated hierarchical patch generation for the liver, right kidney and pancreas. Patches were generated independently for each organ. Note that level 1 patches of the liver (Figure 2) are related to those of the head of the pancreas and superior lobe of the right kidney (Figure 1). For visualization purposes we present the "matching" patches in the same colors: red and light green respectively. Otherwise, patch colors are random. These "matched" patches result from the anatomical relation between liver, pancreas and right kidney (which interact physically with each other). Hence, the automated patching can provide an insight into interorgan interaction and global abdominal deformation.
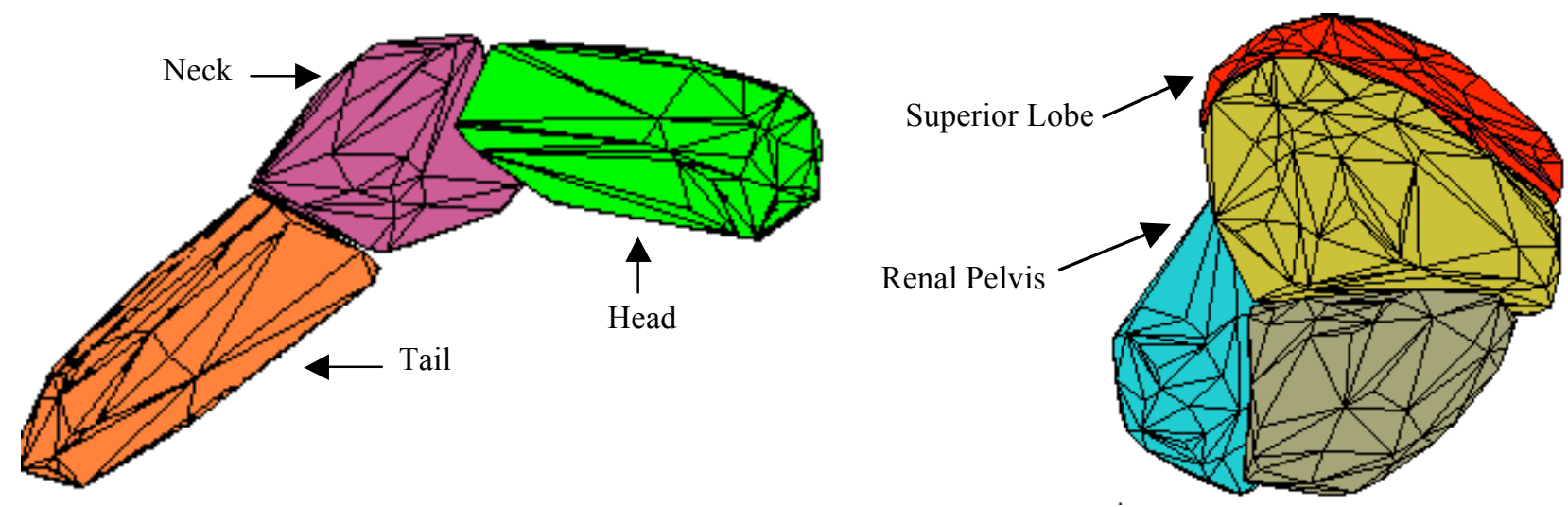

Figure 1: Patch generation for the first mode of anatomical variability/deformations of the pancreas (left) and the right kidney (right) using PFA. Organs are shown in posterior view with patches having different colors.

Statistical shape analysis from organ partitioning further allows the identification and interpretation of the automatically generated patches to understand anatomical and biomechanical characteristics of abdominal organs. In Figure 1.a we observe the correlation between the patches of the first mode and the anatomical boundaries between the head, neck and tail of the pancreas; the head (green) is the best anchored part of the organ and located near the liver, and the tail (orange) the most mobile. The analysis of the right kidney in Figure 1.b shows a first anterior-posterior separation at the location of the renal pelvis and blood vessels; then clusters emphasize the superior pole (red) located against the liver, and the inferior pole (blue) with the abdominal muscles impression. 
Level 1

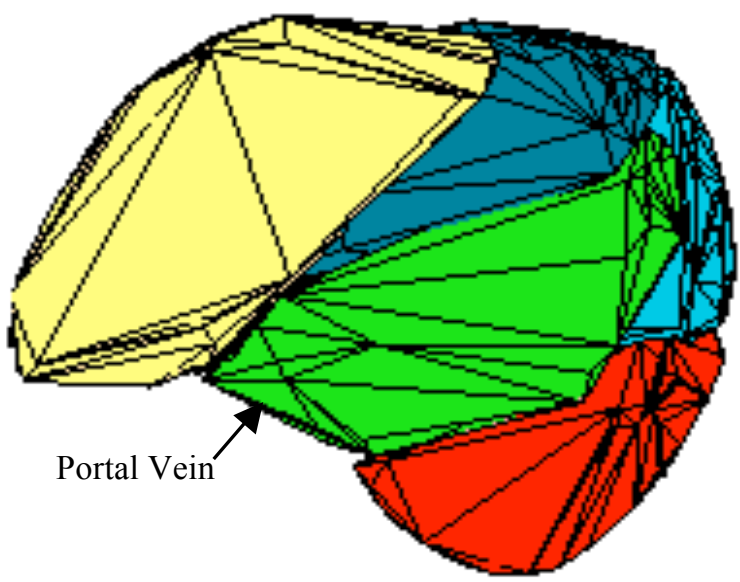

Level 2

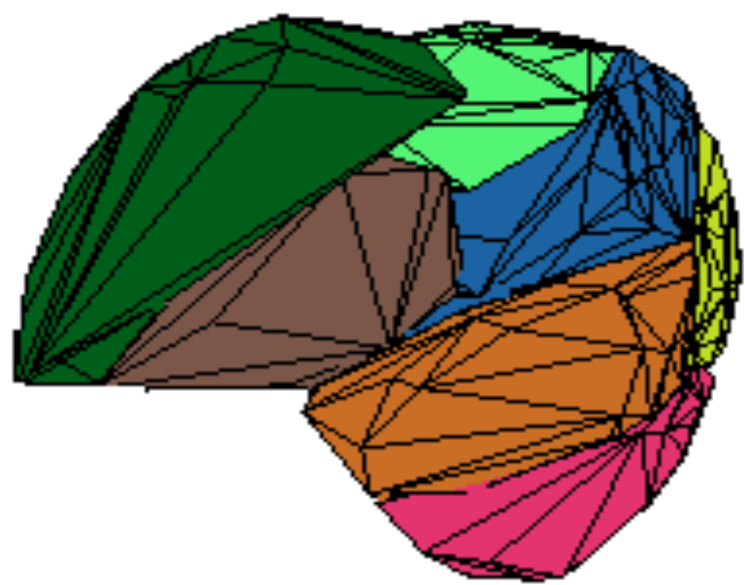

Level 3

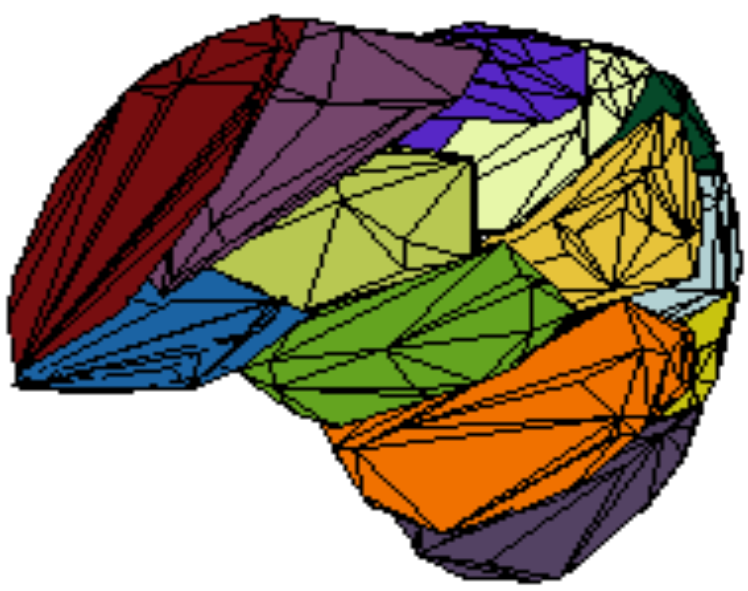

Figure 2: Hierarchical path generation for the first mode of anatomical variability deformations of the liver (in posterior view). Constraints on patch generation are based on the sparseness of the deformations and clusters size and allow the hierarchical patch generation of liver surface (levels 1 to 3 ).

Similarly, the clusters of the first mode of deformation of the liver (Figure 2) present at the coarse level (level 1) a patch for the caudate lobe at the area of high vascularity around the portal vein (green), another for the high structural variability of the left lobe (yellow) and patches associated with the inferior (red), superior (light blue) and medial (dark blue) liver segments. Automatically defined patches may correspond to the popular segmental anatomy of Couinaud liver classification [3]. Hierarchically, finer clustering details are presented at levels 2 and 3 in Figure 2. The hierarchical number of patches reflects the degree of homogeneity of the deformations fields per principal mode.

The clusterizations of the second and third modes of deformation after PFA decomposition are presented in Figure 3. While the patches corresponding to the second mode may be related to the superior, medial and inferior segments, the third mode defines an anterior-posterior separation of the liver. As expected, these results suggest that modes of deformations describe complementary information about anatomical and biomechanical properties of the organs. 

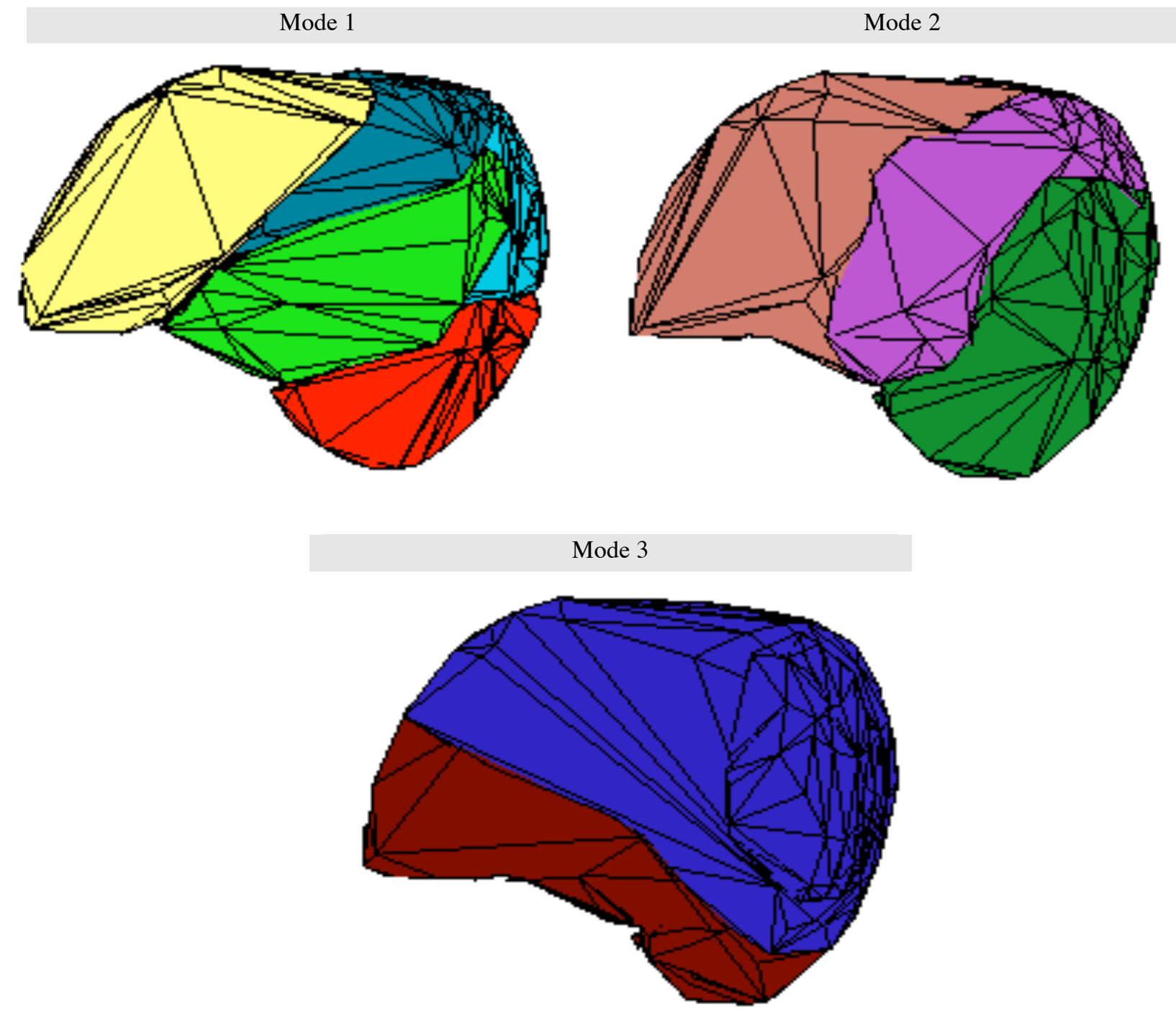

Figure 3: Patch generation for the first three modes of anatomical variability of the/deformation of the liver using PFA.

The anatomical variability analysis is correlated with key anatomical landmarks of the studied organs, which can be input as physical and biomechanical constraints in the analysis of the abdomen. The analysis of the remaining seven studied abdominal organs was also performed, but not presented in this paper due to the limited space.

\section{DISCUSSION}

In this paper we present a method for automated analysis of organ shape variability in the abdomen, based on a combination of statistical atlases, principal factor analysis, and a vector field clustering technique. This shape decomposition scheme allows to model in a compact representation the shape variability found in a training set of images, and it yields intuitive and more interpretable shape decomposition. 
The proposed clusterization technique aimed to quantitatively study the interpretability characteristics of statistical shape models. Using patches of regular or homogeneous patterns of the shape deformation on the surface, our method helps the interpretation of shape variability decomposition. The analysis of deformation fields showed a strong correlation with anatomical landmarks and known mechanical deformations in the abdomen.

The shape decomposition found in our approach resulted in an automatic partitioning of the organ into a set number of patches for each level of detail. The reported patches correspond to popularly-used anatomical segments and show an insight into the possibility to perform automated anatomical reconstruction. Furthermore, the patches at each level were not based on simple subdivision, which may cause problems at the boundaries. Instead, patches at different levels overlap, thus overcoming artifacts imposed by adhesiveness constraints or over-fitting at the boundaries. In contrast, previous work was based on manual definition of these patches with unclear anatomical correlation, which is timeconsuming and non-reproducible.

A clusterization technique was developed for unstructured 3D displacement vector fields across a surface to quantitatively study the interpretability characteristics of statistical shape models. Using patches of regular or homogeneous patterns of the shape deformation on the surface, our method helped the interpretation of shape variability decomposition and is expected to find intuitive organ relations.

The use of this technique as a complement to multi-level statistical shape models $[8,9,13]$ could have important advantages. The shape decomposition found in our approach results in an automatic partitioning of the organ into a set number of patches for each level of detail. The reported patches correspond to popularly-used anatomical segments and show an insight into the possibility to perform automated anatomical reconstruction. In contrast, previous work was based on manual definition of these patches with unclear anatomical correlation, which is time-consuming and nonreproducible $[8,9]$. Further, the patches at each level are not based on simple subdivision, which may cause problems at the boundaries [13]. Instead, patches at different levels overlap, thus overcoming artifacts imposed by adhesiveness constraints or over-fitting at the boundaries.

The work presented is currently being extended to multi-organ shape representations, involving the analysis of areas in organs that have consistent biomechanical deformations. Our approach is expected to find a compact shape variability decomposition that allows for intuitive interpretation of organ relations.

\section{CONCLUSION}

To summarize, we presented a method for automated analysis of organ shape variability in the abdomen, based on a combination of statistical atlases, principal factor analysis, and a vector field clustering technique. This hierarchical shape decomposition scheme allowed to model in a compact representation the shape variability found in a training set of images, and it yielded intuitive and interpretable shape analysis. The evaluation of deformation fields showed a strong correlation with anatomical landmarks and known mechanical deformations in the abdomen. The automated hierarchical partitioning of organs identified anatomically significant components that represent potentially important constraints for abdominal diagnosis and modeling, and that may be used as a complement to multi-level statistical shape models.

\section{ACKNOWLEDGEMNT}

This work was supported in part by the Intramural Research Program of the National Institutes of Health, Clinical Center. 


\section{REFERENCES}

[1] Bishop, C.M. Svensen, M. Williams, C.K.I.: GTM: The Generative Topographic Mapping. In: Obermayer, K. Sejnowski, T.J. (eds.) Self-organizing map formation. MIT Press (2001) 291-310

[2] Cootes, T.F. Edwards, G.J. Taylor, C.J.: Active appearance models. IEEE Transactions on Pattern Analysis and Machine Intelligence, Vol. 23(6) (2001) 681-85

[3] Couinaud C. [The anatomy of the liver] Ann Ital Chir. Vol. 63(6) (1992 ) 693-7 (French)

[4] Fodor, I.: A survey of dimension reduction techniques. Lawrence Livermore National Laboratory Technical Report no. UCRLID-148494 (2002)

[5] Gonzalez Ballester, M.A Linguraru, M.G. Reyes Aguirre M. Ayache, N.: On the Adequacy of Principal Factor Analysis for the Study of Shape Variability. In: Fitzpatrick, J.M. and Reinhardt, J.M. (eds.): SPIE Medical Imaging 2005: Image Processing, Vol. 5747 (2005) 1392-1399

[6] Kaiser, H. F.: The Varimax Criterion for Analytic Rotation in Factor Analysis. Psychometrika, Vol. 23(3) (1958) 187-200

[7] Linguraru, M.G. Sandberg, J.K.Li, Z. Pura, J.A. Summers, R.M.: Atlas-based Automated Segmentation of Spleen and Liver using Adaptive Enhancement Estimation. In: Yang, G.Z. et al. (eds.) Medical Image Computing and Computer-Assisted Intervention MICCAI 2009, Lecture Notes in Computer Science, Vol. 5762, Springer New York (2009) 1001-1008

[8] Okada, T. Yokota, K. Hori, M. Nakamoto, M. Nakamura, H. Sato, Y.: Construction of Hierarchical Multi-Organ Statistical Atlases and their Application to Multi-Organ Segmentation from CT Images. In: Metaxas, D. et al. (Eds.): MICCAI 2008, Part I, LNCS 5241 (2008) 502-509

[9] Okada, T. Shimada, R. Hori, M. Nakamoto, M. Chen, Y-W. Nakamura, H. Sato, Y. Automated Segmentation of the Liver from 3D CT Images using Probabilistic Atlas and Multilevel Statistical Shape Models. Academic Radiology, Vol. 15 (2008) 1390-1403

[10] Roy, T. et al.: Segmentation of a vector field: dominant parameter and shape optimization. Journal of Mathematical Imaging and Vision, Vol. 24(2) (2006) 259-76

[11] Rubin, D. B., Thayer, D. T.: EM Algorithms for ML Factor Analysis. Psychometrika, Vol. 47(1) (1982) 69-76

[12] Rueckert, D. et al.: Non-rigid registration using free-form deformations: Application to breast MR images. IEEE Transactions on Medical Imaging, Vol. 18(8) (1999) 712-21

[13] Syrkina, E. González Ballester, M.A. Székelym G.: Correspondence Establishment in Statistical Modelling of Shapes with Arbitrary Topology. IEEE Workshop on Mathematical Methods for Biomedical Image Analysis (MMBIA) (2007)

[14] Studholme, C. Hill, D.L.G. Hawkes, D.J.: An overlap invariant entropy measure of 3D medical image alignment. Pattern Recognition, Vol. 32(1) (1999) 71-86 